\title{
Intrathecal Cefazolin-Induced Seizures Following ATTEMPTED DISCOGRAPHY
}

\author{
Mark V. Boswell, MD, PhD, and James R. Wolfe, MD
}

This report describes a 39 year-old woman who underwent attempted discography and intradiscal electrothermal therapy (IDET) of the L5/S1 intervertebral disc. The procedure was abandoned after multiple unsuccessful attempts to cannulate the disc. The case was complicated by at least two lumbar dural punctures, confirmed by injection of nonionic contrast that contained 12.5 $\mathrm{mg} / \mathrm{mL}$ of cefazolin, included for prophylaxis of discitis. About 45 minutes later the patient developed severe back pain. Shortly thereafter she became progressively agitat- ed and confused, and developed intractable seizures and coma. Despite aggressive treatment the patient could not be resuscitated and expired several hours later.

Convulsions were initially attributed to an adverse reaction to meperidine and promethazine, given for the back pain, however this explanation proved to be untenable. In addition, the accidental administration of an ionic contrast agent, such as Hypaque ${ }^{\circledR}$, was excluded. Based on a detailed review of the case and the literature, it was concluded that the patient succumbed from an unintention- al dose of intrathecal cefazolin, which had been diluted in the nonionic contrast agent that was used to confirm needle placement. Available evidence indicates that cefazolin is a potent epileptogenic agent when given intrathecally. The facts of the case and the evidence supporting the conclusion are presented. It is recommended that cefazolin not be mixed with the contrast agent used to document initial needle placement during discography.

Keywords: Cefazolin, intrathecal, discography, seizures
Discography is commonly used for the diagnosis of low back pain. The most serious complication of discography is considered to be discitis. The incidence of discitis may be in the range of $5 \%$ without the use of prophylactic antibiotics (1). The reported incidence falls to approximately 0.1 to $0.2 \%$ when antibiotics are used (2). Indeed, the severity of the complication prompts most clinicians to administer prophylactic antibiotics, typically a cephalosporin given systemically and/or intradiscally. When giving the intradiscal antibiotic, many physicians mix cefazolin with a nonionic contrast agent, typically Omnipaque ${ }^{\circledR}$. Based on personal preference, the final concentration of cefazolin in the contrast agent may range from $1 \mathrm{mg} / \mathrm{mL}$ to $10 \mathrm{mg} / \mathrm{mL}$ or higher, depending on the technique used to dilute the antibiotic.

From Case School of Medicine and University Hospitals of Cleveland, Cleveland, Ohio and MedCentral Health System, Mansfield, Ohio. Address Correspondence: Mark V. Boswell, MD, PhD, Department of Anesthesiology, University Hospitals, 11100 Euclid Avenue, Cleveland, Ohio 44106.

E-mail: mark.boswell@uhhs.com

Support:There was no external funding in preparation of this manuscript

Conflict of Interest: None
Physicians are generally comfortable giving cefazolin to patients who are not allergic to the medication. This is especially true with anesthesiologists, who are used to injecting the drug intravenously prior to starting an anesthetic; the drug is considered to be essentially nontoxic. However, few physicians are aware that betalactam antibiotics can be toxic when instilled intrathecally, with a propensity to cause seizures. This property is shared by penicillin derivatives and cephalosporins.

This report describes the case of a patient who developed intractable seizure-like activity shortly after returning to the recovery area following an attempted discogram and intradiscal electrothermal therapy (IDET) procedure. The procedure was abandoned after multiple unsuccessful attempts to cannulate the disc. The case was complicated by at least two lumbar dural punctures, confirmed by injection of nonionic contrast that contained $12.5 \mathrm{mg} / \mathrm{mL}$ of cefazolin, included for the prophylaxis of discitis. The convulsions were initially attributed to meperidine and promethazine administered for post-procedure pain. An accidental intrathecal administration of nonionic contrast containing cefazolin was not considered by the treating physicians in the differential diagnosis of the seizures.
Despite timely and aggressive efforts, including full resuscitative measures, the seizures could not be reversed and the patient expired several hours later. The severity of the complication, potential mechanisms involved and implications for interventional pain physicians prompt the presentation of this case.

\section{Presentation of Case}

The patient was a 39 year-old woman with low back pain who was admitted to the hospital for intradiscal electrothermal therapy. She had a history of prior discectomy with initial benefit, but pain subsequently returned. Diagnostic injections, including epidural steroid injections and facet injections were not helpful. A three level discogram performed three weeks earlier confirmed discogenic pain arising from the L5-S1 disc, whereas the L3-4 and L4-5 discs were unremarkable.

The patient was taken to the procedure room at 13:00 hrs. After informed consent, she was placed in the prone position, her back was prepped and draped in a sterile fashion and lidocaine was used to anesthetize the skin. Continuous noninvasive monitoring was used throughout the case, including BP, pulse rate, and pulse oximetry. Vital signs remained stable, with $\mathrm{O}_{2}$ saturations from $96 \%$ to $98 \%$. 
Midazolam was administered in increments of $2 \mathrm{mg}$ (a total of $8 \mathrm{mg}$ ) for sedation between 13:10 hours and 13:23. Under fluoroscopic guidance, five attempts were made using different angles on the fluoroscope to enter the L5-S1 disc, but without success. In the process, there were two lumbar dural punctures, which were confirmed with test doses of Omnipaque containing cefazolin. The Omnipaque was prepared by diluting $250 \mathrm{mg}$ of cefazolin with $20 \mathrm{~mL}$ of Omnipaque (12.5 $\mathrm{mg} / \mathrm{mL}$ of cefazolin). Approximately 2 to $3 \mathrm{~mL}$ of the contrast-antibiotic mixture was injected. The resultant myelogram demonstrated intrathecal contrast. However, the intradiscal space was never adequately outlined. After discussion with the patient, the decision was made to stop the procedure. Because there had been two definite dural punctures, a prophylactic epidural blood patch was performed without difficulty. The procedure concluded at 14:05 hours. The patient was able to move off of the procedure table under her own power, did not have procedural back pain at that time, and was returned to short term care for recovery.

However, on arrival in the recovery area, the patient was complaining of severe back pain. The first set of vital signs, recorded at 14:20 hours were as follows: blood pressure $132 / 86$, pulse 80 per minute and respiratory rate of 16 breaths per minute. Meperidine (Demerol®) $75 \mathrm{mg}$ and promethazine (Phenergan $\left.{ }^{\circledR}\right) 25 \mathrm{mg}$ were administered intravenously over several minutes at 14:30 hours. The patient remained in pain, vomited and became disoriented, attempting to crawl out of bed at 14:50 hours.

Several minutes later she pulled out her IV. She began to have what was described as seizure activity at 14:58 hours, with jerking movements and she became unresponsive. She continued to have dystonic movements, with jerking of her arms and legs. Pupils were dilated and she was noted to be foaming at the mouth. She became tachycardic and developed left sided facial twitching and some arm stiffness. The $\mathrm{O}_{2}$ saturation on room air was $93 \%$ at $15: 03$ hours.

A code was called at that time and the resuscitation team arrived promptly. The differential diagnoses were seizures due to meperidine or a dystonic reaction from promethazine. Diphenhydramine (50 $\mathrm{mg})$, naloxone $(0.4 \mathrm{mg}$ ) and lorazepam $(1 \mathrm{mg})$ were administered with no effect on the seizure activity; additional doses of lorazepam, diphenhydramine and midazolam were administered, again with no effect. The patient never stopped breathing spontaneously. To protect the airway, atracurium was used to facilitate intubation, mechanical ventilation was instituted, and she was transferred to the intensive care unit, where she was placed on the ventilator. Resuscitative efforts were continued. An arterial blood gas, on the ventilator at 16:20 hrs with an $\mathrm{FIO}_{2}$ of $50 \%$ was: $\mathrm{pH} 7.15, \mathrm{PCO}_{2} 46, \mathrm{O}_{2} 84, \mathrm{HCO}_{3}$ 16 and base excess -12.8 . Intravenous fosphenytoin was administered without effect. The patient's condition worsened despite aggressive therapy. A subsequent arterial blood gas, at 18:25 hours, was: $\mathrm{pH}$ 7.00, $\mathrm{CO}_{2} 66, \mathrm{O}_{2} 77, \mathrm{HCO}_{3} 16$ and $\mathrm{BE}-$ 15.6. Shortly thereafter, the patient developed ventricular tachycardia and became pulseless and asystolic. Cardiopulmonary resuscitation (CPR) was instituted according to the advanced cardiac life support (ACLS) protocol and a transvenous pacemaker was inserted. Defibrillation and prolonged CPR were unsuccessful and the patient expired at 18:48 hours, having never regained consciousness.

Autopsy by the county coroner failed to demonstrate any significant abnormalities, other than mild brain edema on microscopic brain sections. Examination of the lungs showed only mild alveolar hemorrhage but disclosed no evidence of allergic reaction. The heart was normal. Ante-mortem meperidine and normeperidine levels were in the nontoxic range. Spinal fluid drug levels were not obtained. No anatomic or pathologic cause of death could be identified, and the coroner determined that the patient died of an adverse drug interaction.

\section{DISCUSSION}

The patient in this case presented for a routine IDET procedure. The cause of her death was unclear to the treating physicians at the time of the incident. The family ultimately filed a lawsuit, and the medical records were sent by the defense attorney for review. On initial examination of the records, it was noted that the diagnoses by the treating physicians focused on meperidine and promethazine, drugs known to cause seizures or dystonic reactions, respectively. However, the doses were too low to explain the sudden and irreversible deterioration of the patient. Drug levels were not in the tox- ic range. Importantly, the patient never stopped breathing spontaneously until intubated and placed on the ventilator, which excluded an unrecognized overdose of opioid.

The hypothesis that the events described here were due to an adverse reaction to meperidine or promethazine was untenable. Meperidine can be given safely to patients with normal renal function up to a total dose of $600 \mathrm{mg}$ in 24 hours, for up to 48 hours (3). This patient had one dose of $75 \mathrm{mg}$, and ante-mortem blood levels were not in the toxic range (meperidine $25 \mathrm{mcg} / \mathrm{dl}$ and normeperidine $7 \mathrm{mcg} / \mathrm{dl}$ ). Although promethazine is a phenothiazine, prolonged severe seizures are not possible with such a small dose; the ante-mortem promethazine blood level was in the therapeutic range (1.0 $\mathrm{mcg} / \mathrm{dl}$ ). Neither the treating physicians or the coroner considered the possibility that the reaction was due to the intrathecal injection of a medication, although at lease two dural punctures were documented in the chart, and accidental dural puncture is a recognized complication of discography (2).

This prompted a detailed review of all medications given. It was concluded that only two possible medications could have explained the scenario described, the contrast agent or the antibiotic. The possibility that an ionic contrast agent such as diatrizoate meglumine (Hypaque ${ }^{\circledR}$ ) was given by accident, instead of Omnipaque, was considered. Intrathecal Hypaque is known to cause seizures when given intrathecally (4). However, this possibility was definitely excluded. This prompted a literature search on the possible effects of intrathecal cefazolin, which was known to have been administered intrathecally with the Omnipaque. Several important articles were identified, and are discussed.

Lang et al (5) published a case report of a massive intrathecal dose of cefazolin given after a neurosurgical case. The report was in the European Journal of Anesthesiology, which is not readily available to most physicians in the United States. That case described the clinical course of a patient who received a large dose of cefazolin unintentionally through the lumbar drain, which had been placed in conjunction with a trans-sphenoidal hypophysectomy. Ten minutes after the administration of $1.5 \mathrm{~g}$ of cefazolin into the lumbar drain, the patient complained of bilateral sciatica. She soon became diaphoret- 
ic and nauseated, pain increased and she developed generalized tonic-clonic seizures and bilateral dilated pupils. After intubation, the seizures continued; ultimately they were controlled with phenobarbitone coma, diazepam and cisatracurium. Initial CSF levels of cefazolin were $3.7 \mathrm{mg} / \mathrm{mL}$ shortly after the unintentional dose. $600 \mathrm{~mL}$ of CSF was collected from the drain over the next 24 hours. Sedation and analgesia were tapered over the next 3 days and on day 4 she was extubated. The patient made a slow recovery over the next 15 months.

Although the dose was extremely high in that case, the clinical course was similar to the present case, with unexplained onset of back pain followed by nausea, seizures, dilated pupils and unresponsiveness. The time course was consistent with ascending CSF circulation and cefazolin ultimately reaching the brain, causing seizures and unresponsiveness.

In the present case, the dose of cefazolin was obviously much lower than the neurosurgical case, but similarities in the clinical course were evident. Calculation of initial CSF cefazolin levels, assuming that about $25 \mathrm{mg}$ of antibiotic was administered with the Omnipaque $(2 \mathrm{~mL} x$ $12.5 \mathrm{mg} / \mathrm{mL}$ ) into approximately $100 \mathrm{~mL}$ of CSF, would yield an initial CSF concentration of $0.25 \mathrm{mg} / \mathrm{mL}$, about 10 times lower than the initial concentration in the neurosurgical case described above. The question remained as to whether this concentration was sufficient to cause the adverse reaction. A review of the literature disclosed that a CSF cefazolin level of 34 $\mathrm{mcg} / \mathrm{mL}(0.034 \mathrm{mg} / \mathrm{mL})$ caused seizures in a patient given the antibiotic intravenously (6). Seizures also have been reported after intraventricular administration of cefazolin (7). Thus, these observations strongly suggest that the seizure activity and demise of the patient described here were due to unintentional intrathecal administration of cefazolin.

The epileptogenic activities of betalactam antibiotics have been studied in rats (8). Cefazolin was the most potent epileptogenic compound tested, about 3 times more potent than benzylpenicillin, and much more potent than other cephalosporins, including ceftriaxone, cefoperazone and cefamandole.

The mechanism of seizure activity of beta-lactam antibiotics has been determined. Available evidence clearly demonstrates that the mechanism of cephalo- sporin-induced convulsions is mediated mainly by inhibition of GABA (A) receptor activity and not through NMDA receptor modulation (9). Thus, cefazolin can cause massive depolarization of neurons in the central nervous system, causing seizures and coma. It is noteworthy that pathologic examination would not be expected to detect an abnormality, because the effect is electrophysiologic and not structural. This mechanism would explain the progressive acidosis and hypercarbia seen with the patient described here, despite increasing rates of mechanical ventilation. Encephalopathy due to transient hypoxemia or an adverse reaction to meperidine or promethazine could not have caused this progressive and irreversible sequence of events. Indeed, only mild brain edema, and no evidence of encephalopathy was found on autopsy. Depending on the dose of intrathecal cefazolin administered, without immediate efforts to control seizures, including barbiturate coma with EEG burst suppression, tracheal intubation, sustained muscle relaxation to prevent metabolic acidosis, and drainage of CSF, the outcome may be poor.

A comment should be made about the outcome of the trial resulting from this case. The jury found in favor of the defendant, noting that dural puncture is a recognized complication of discography and that intradiscal injection of cefazolin is accepted medical practice. Despite the tragic outcome, the jury found that the actions of the physician did not deviate from the known standards of medical care at that time and dismissed the case. The jury also discounted the allegation made by plaintiff that the patient's death was due to an adverse drug reaction involving meperidine and promethazine.

Based on this case, several recommendations may be made. Physicians should not mix cefazolin with the contrast agent used for initial verification of needle placement. Only after intradiscal placement of the needle is confirmed by contrast injection should intradiscal antibiotic be given. It is prudent to use the lowest dose possible and to be certain that dural puncture did not occur before giving the antibiotic. It should be noted that cefazolin is probably the worst antibiotic to use, from the standpoint of its epileptogenic potential. Based on animal studies, ceftriaxone, an antibiotic used frequently in neurosurgical cases, theoretically may be safer than cefazolin.

\section{ConcLusion}

The case presented here appears to be the first report of post-discography seizures associated with intrathecal cefazolin administration. Likely explanations for the sequence of events described herein, including unintentional Hypaque administration, or adverse drug reaction involving meperidine and promethazine, were excluded to a reasonable degree of medical certainty. The risk of discitis, the most feared complication of discography, argues for the use of prophylactic antibiotics, either systemically or intradiscally. Cefazolin appears to be the most commonly used antibiotic for this purpose. However, the potential risk of unintentional dural puncture and accidental administration of a toxic dose of intrathecal cefazolin should not be overlooked. Consideration should be given to using another cephalosporin, with less toxic potential, such as ceftriaxone. If an intradiscal injection of antibiotic is given, a low dose should be used, and then only when the dose is certain not to go into the CSF. In this regard, it is recommended that the antibiotic not be mixed with the contrast agent used to verify initial needle placement.

\section{Author Affiliation: \\ Mark V. Boswell, MD, PhD \\ Associate Professor of Anesthesiology \\ Chief, Pain Medicine Service \\ Director, Pain Medicine Fellowship \\ Department of Anesthesiology \\ Case School of Medicine and \\ University Hospitals of Cleveland \\ 11100 Euclid Avenue \\ Cleveland, Ohio 44106 \\ E-mail: mark.boswell@uhhs.com \\ James R. Wolfe, MD \\ Medical Director, PainCare \\ MedCentral Health System \\ 1020 Cricket Lane \\ Mansfield, Ohio 44906 \\ E-mail: jwolfe@medcentral.org}

\section{REFERENCES}

1. Osti OL, Fraser RD, Vernon-Roberts B. Discitis after discography: The role of prophylactic antibiotics. J Bone Joint Surg Br 1990; 72:271-274.

2. Abdi S, Zhou Y, Patel $\mathrm{N}$ et al. Lumbar discography. In Manchikanti L, Slipman CW, Fellows B (eds). Low Back Pain. ASIPP Publishing, Paducah, 2002; 249-256, 
3. American Pain Society. Principles of Analgesic Use in the Treatment of Acute Pain and Cancer Pain. Fifth edition. APS, Glenview, 2003; 36.

4. Killeffer JA, Kaufman HH. Inadvertent intraoperative myelography with Hypaque: case report and discussion. Surg Neurol 1997; 48:70-73.

5 Lang EW, Weinert D, Behnke A et al. A massive intrathecal cefazolin overdose. Case report. Eur J Anaesthesiology 1999;
16:204-205.

6. Bechtel TP. Slaughter RL, Moore TD. Seizures associated with high cerebrospinal fluid concentrations of cefazolin. Am J Hosp Pharm 1980; 37:271-273.

7. Martin ES III, Bagwell JT, bush-Veith S et al. Seizures after intraventricular cefazolin administration. Case report. Clin Pharm 1992; 11:104-105.

8. De Sarro A, Ammendola D, Zappala M et al. Relationship between structure and convulsant properties of some beta-lactam antibiotics following intracerebroventricular microinjection in rats. Antimicrob Agents Chemother 1995; 39:232-237.

9. Sugimoto M, Uchida I, Mashimo T et al. Evidence for the involvement of GABA (A) receptor blockade in convulsions induced by cephalosporins. Neuropharmacology 2003; 45:304-314. 